\title{
Neutral Hydrogen in the Local Universe
}

\author{
D. Obreschkow
}

Astrophysics, Department of Physics, University of Oxford, Keble Road, Oxford, OX1 3RH, UK

\begin{abstract}
We revisit the composition of neutral hydrogen, i.e. atomic (HI) and molecular $\left(\mathrm{H}_{2}\right)$, in the local Universe, with focus on the $\mathrm{H}_{2}$-to-HI mass ratio $\eta_{\text {gal }}$ in various galaxies and the $\mathrm{H}_{2}$-mass function (MF). First, we derive the $\mathrm{H}_{2}-\mathrm{MF}$ from the CO-luminosity function (LF) of the FCRAO Extragalactic CO Survey, using a variable CO-to- $\mathrm{H}_{2}$ conversion fitted to nearby observations. This implies a universal $\mathrm{H}_{2}$ density $\Omega_{\mathrm{H}_{2}}=(6.9 \pm 2.7) \cdot 10^{-5} h^{-1}$ and $\Omega_{\mathrm{H}_{2}} / \Omega_{\mathrm{HI}}=0.26 \pm 0.11$, which could, however, suffer from incompleteness of the CO-sample. Therefore, a second approach derives the $\mathrm{H}_{2}-\mathrm{MF}$ from a HI-sample with well described completeness (HIPASS). This premisses an estimation of $\eta_{\text {gal }}$, for which we introduce a set of phenomenological models based on a sample of 245 galaxies drawn from the literature. Our best model in terms of statistical scatter describes $\eta_{\text {gal }}$ as a function of galaxy type and cold gas mass. This model leads to an $\mathrm{H}_{2}-\mathrm{MF}$ that matches the one derived from the CO-LF, thus supporting that model and settling completeness uncertainties.
\end{abstract}

Keywords: ISM, Cold gas, Hydrogen, Molecular gas

PACS: 98.58, 67.63, 67.80

\section{INTRODUCTION}

Neutral hydrogen in galaxies coexists in the atomic (HI) and molecular phase $\left(\mathrm{H}_{2}\right)$. Most $\mathrm{H}_{2}$-mass estimations rely on indirect tracers with uncertain conversion factors. In consequence, the mass ratio $\eta \equiv d \mathrm{M}_{\mathrm{H}_{2}} / d \mathrm{M}_{\mathrm{HI}}$ and its value for individual galaxies $\eta_{\mathrm{gal}} \equiv \mathrm{M}_{\mathrm{H}_{2}} / \mathrm{M}_{\mathrm{HI}}$ remain uncertain, and estimates of the universal average $\eta_{\mathrm{u}}=\Omega_{\mathrm{H}_{2}} / \Omega_{\mathrm{HI}}$ vary by an order of magnitude at $z=0$ (e.g. 0.14 [1], 0.42 [2], 1.1 [3]). This issue culminates in the comparison of simulated cold gas-MFs with observed HI-MFs, as illustrated in Fig. 1 showing various HI-MFs derived from two simulated cold gas-MFs.

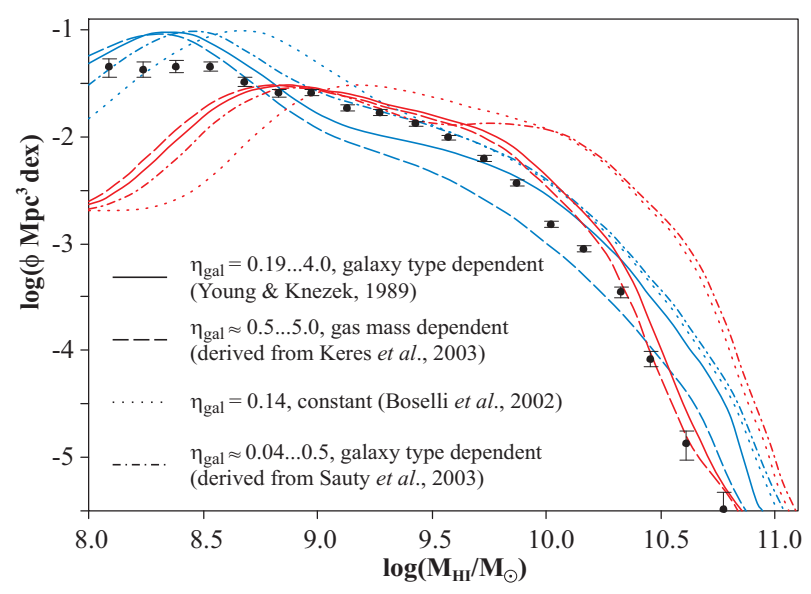

FIGURE 1. Observed HI-MF [6] (points) vs simulated HI-MFs derived from two semi-analytic models [7] (red) and [8] (blue) for recent models of $\eta_{\text {gal }}$. All plots adopt $H_{0}=73 \mathrm{~km} \mathrm{~s}^{-1} \mathrm{Mpc}^{-1}$. 
This proceeding aims at unifying recent studies of the HI-MF, CO-LF, CO-to- $\mathrm{H}_{2}$ conversion ( $X$-factor), and $\eta_{\text {gal }}$ in various galaxies. In Sect. 2, we recover the $\mathrm{H}_{2}-\mathrm{MF}$ from the CO-LF [2]. Uncertainties caused by the incompleteness of the underlying sample are bypassed in Sect. 3, where we construct the $\mathrm{H}_{2}-\mathrm{MF}$ from the HIPASS HIsample with well characterized completeness. As this approach premisses a model of $\eta_{\text {gal }}$, which itself relies on $\mathrm{H}_{2}$-estimations from $\mathrm{CO}$, both approaches ultimately depend on the $X$-factor, for which we use a galaxy dependent model.

\section{2. $\mathrm{H}_{2}$-MF FROM CO DATA}

\subsection{CO-to-H $\mathrm{H}_{2}$ Conversion Revisited}

Most $\mathrm{H}_{2}$-mass estimations rely on indirect detections of the CO-rotation line at $115 \mathrm{GHz}$, which is powered by $\mathrm{H}_{2}-\mathrm{CO}$ collisions and hence dependent on the $\mathrm{H}_{2}$-mass $\mathrm{M}_{\mathrm{H}_{2}}$, the ratio $\mathrm{M}_{\mathrm{H}_{2}} / \mathrm{M}_{\mathrm{CO}}$, and pressure. $\mathrm{CO}$ is optically thick for this radiation; thus the $115 \mathrm{GHz}$ rest frame luminosity $L_{115}$ is a non-linear mass tracer for individual clouds. However, detailed studies revealed that on kiloparsec scales (hundreds of clouds) CO is effectively thin $[9,10]$, in the sense that $L_{115}$ is nearly proportional to $\mathrm{M}_{\mathrm{H}_{2}}$, or more precisely [11],

$$
\frac{\mathrm{M}_{\mathrm{H}_{2}}}{\mathrm{M}_{\odot}}=5.37 \cdot 10^{3} \cdot X \cdot \frac{S_{115}}{\mathrm{Jy} \mathrm{km} \mathrm{s}^{-1}} \cdot\left(\frac{D_{l}}{\mathrm{Mpc}}\right)^{2}=1.17 \cdot 10^{-22} \cdot X \cdot \frac{L_{115}}{W}
$$

where $S_{115}$ is the integrated line flux and $D_{l}$ is the luminosity distance. In this equation, the dimensionless ratio $X \equiv\left[N\left(\mathrm{H}_{2}\right) / \mathrm{cm}^{-2}\right] /\left[I_{115} /\left(\mathrm{K} \mathrm{km} \mathrm{s}^{-1}\right)\right] \cdot 10^{-20}$ is assumed constant for identical average cloud properties (geometry, metallicity, pressure).

The observational determination of $X$ requires $\mathrm{CO}$-independent $\mathrm{H}_{2}$-measurements, which are currently restricted to a few nearby galaxies. Typical methods use the virialmass of giant molecular clouds [12], the line ratios of different $\mathrm{CO}$ isotopes [13], the gas-to-dust ratio $[14,15]$, or $\gamma$-ray data [16]. Based on early findings that $X$ is constant in the inner $2-10 \mathrm{kpc}$ of the Galaxy, several authors (e.g. [12]) concluded that $X$ does not depend on average cloud properties. This assertion was later rejected [17, 1], when larger samples pinpointed a clear correlation between $X$ and the average metallicity.

We use a variable $X$-factor $X_{v}$ depending on the extinction corrected absolute blue magnitude $M_{\mathrm{B}}$ or the rest frame $115 \mathrm{GHz}$-luminosity $L_{115}$. These relations are almost as tight as the metallicity-dependent one, and we prefer them for the widespread availability of $M_{\mathrm{B}}$-data and the implicit access to $L_{115}$. The observational data (Fig. 2, left) was drawn from the literature and the best linear fit minimizing the square deviations while respecting the relation between $L_{115}$ and $M_{\mathrm{B}}$ (based on the 245 galaxies in $\S 3.1$ ) is,

$$
\log \left(X_{v}\right)=8.63-0.309 \log \left(L_{115} h^{2} \mathrm{~W}^{-1}\right) \pm \sigma_{X}=3.35+0.159\left(M_{\mathrm{B}}-5 \log h\right) \pm \sigma_{X}
$$

Both relations have the same rms-scatter of 0.29 in $\log (X)$, which implies a physical scatter of $\sigma_{X}=0.26$ when accounting for the observational noise of 0.13 dex. To compare the following results to the ones obtained with a constant $X$-factor, we will also use a constant model $\log \left(X_{c}\right)=\log (2) \pm \sigma_{X}$ [25] with the same scatter. 

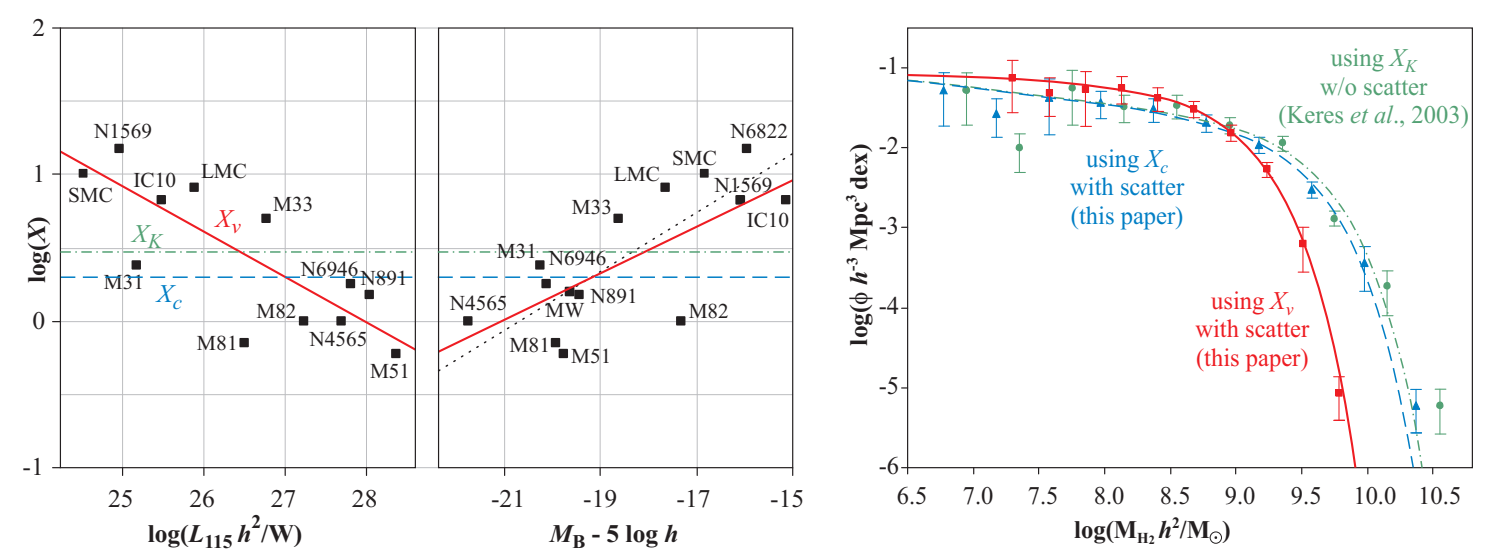

FIGURE 2. LEFT: (Points) Measured $X$-factor as a function of $115 \mathrm{GHz}$ luminosity $L_{115}$ and absolute blue magnitude $M_{\mathrm{B}}$ for 14 local galaxies [1, 18, 1, 19, 20, 21, 22, 23, 24], (red solid lines) our best variable model $X_{v}$, (blue dashed lines) our constant choice $X_{c}$, (green dash-dotted lines) constant choice by [2], (dotted line) fit by [1]. RIGHT: $\mathrm{H}_{2}$-MFs derived from the CO-LF presented by [2], using the three models for the $X$-factor shown on the left; $X_{v}$ and $X_{c}$ were applied with scatter, $X_{K}$ without scatter.

TABLE 1. Schechter parameters and implied densities. $\eta_{\mathrm{u}}$ uses $\Omega_{H I}=(2.6 \pm 0.3) \cdot 10^{-4} h^{-1}[6]$.

\begin{tabular}{|c|c|c|c|c|c|c|c|}
\hline & $\frac{\mathrm{M}^{*}}{10^{9} h^{-2} \mathrm{M}_{\odot}}$ & $\alpha$ & $\frac{\theta}{h^{3} \mathrm{Mpc}^{-3} \mathrm{dex}^{-1}}$ & Red. $\chi^{2}$ & $\frac{\rho_{\mathrm{H}_{2}}}{10^{7} h \mathrm{M}_{\odot} \mathrm{Mpc}^{-3}}$ & $\frac{\Omega_{\mathrm{H}_{2}}}{10^{-4} h^{-1}}$ & $\eta_{\mathrm{u}}$ \\
\hline$X_{c}$ & 2.43 & -1.20 & 0.0082 & 0.34 & $2.3 \pm 0.9$ & $0.83 \pm 0.31$ & $0.32 \pm 0.13$ \\
$\mathbf{X}_{\mathbf{v}}$ & $\mathbf{0 . 7 5}$ & $-\mathbf{1 . 0 7}$ & $\mathbf{0 . 0 2 4 3}$ & $\mathbf{0 . 0 5}$ & $\mathbf{1 . 9} \pm \mathbf{0 . 7}$ & $\mathbf{0 . 6 9} \pm \mathbf{0 . 2 7}$ & $\mathbf{0 . 2 6} \pm \mathbf{0 . 1 1}$ \\
$X_{K}$ & 2.81 & -1.18 & 0.0089 & 2.55 & $2.8 \pm 1.1$ & $1.0 \pm 0.39$ & $0.39 \pm 0.16$ \\
\hline
\end{tabular}

\section{2. $\mathrm{H}_{2}$-MF from CO-LF}

We shall now extract $\mathrm{H}_{2}$-MFs from the CO-LF presented by [2]. The latter is based on a far infrared-selected subsample (200 galaxies) of the FCRAO Extragalactic CO Survey [26], which successfully reproduced the $60 \mu \mathrm{m}-\mathrm{LF}$, despite its poorly understood completeness. We first converted the luminosity units used in [2] to Watts (1 $\mathrm{Jy} \mathrm{km} \mathrm{s}^{-1} \mathrm{Mpc}^{2}$ is $3.65 \cdot 10^{24} \mathrm{~W}$ at $115 \mathrm{GHz}$ ) and then transformed the CO-LF to a $\mathrm{H}_{2}-\mathrm{MF}$ via relation (1), right. For the $X$-factor we used the two models introduced above (i.e. the constant value $X_{c}$ and the variable value $X_{v}$, both with scatter) and the constant value $X_{K}=3$ without scatter used by [2] to derive the first $\mathrm{H}_{2}-\mathrm{MF}$.

The resulting $\mathrm{H}_{2}$-MFs are displayed in Fig. 2 together with the Schechter functions minimizing the weighted square deviations. Function parameters and resulting gas densities are listed in Table 1 . For the constant $X$-factor, the change from the $\mathrm{H}_{2}-\mathrm{MF}$ by [2] to our model is small, the total density $\rho_{\mathrm{H}_{2}}$ decreasing by about $19 \%$. This is the combined effect of a mass-shift $X_{c} / X_{K}=0.67$ and the addition of scatter, which slightly raises the high-mass end, thus reincreasing $\rho_{\mathrm{H}_{2}}$ by a factor 1.22 . However, our variable $X$-factor significantly compresses the MF and $\rho_{\mathrm{H}_{2}}$ drops by $33 \%$. In this preferred $\mathrm{H}_{2}-\mathrm{MF}$, the global $\mathrm{H}_{2}$-to-HI ratio equals $26 \pm 11 \%$, implying a cold gas density $\Omega_{\mathrm{g}}=(4.5 \pm 0.8) \cdot 10^{-4} h^{-1}$ composed of $59 \pm 6 \% \mathrm{HI}, 15 \pm 6 \% \mathrm{H}_{2}, 24 \%$ Helium, $2 \%$ Metals (uncertainties of $\mathrm{HI}$ and $\mathrm{H}_{2}$ are anti-correlated). 


\section{3. $\mathrm{H}_{2}$-MF FROM COMBINED HI+CO DATA}

In this section, we derive phenomenological models for the galaxy-averaged molecular ratio $\eta_{\text {gal }}$. All models will be applied to recover $\mathrm{H}_{2}$-MFs from the complete HI-data in HIPASS, thus providing an alternative to using the CO-LF.

\subsection{Molecular-to-Atomic Gas in Different Galaxies}

Our sample of 245 distinct galaxies (presented in [27]) was drawn from the literature $[28,29,30,5,31,32,23,33,34,4,18]$ and includes homogenized CO-line fluxes, HImasses, galaxy types $T$, and extinction corrected absolute B-band magnitudes $M_{\mathrm{B}}$. The latter served to determine the variable $X$-factor $X_{v}$ (eq. 2), used to convert CO-fluxes in $\mathrm{H}_{2}$-masses (eq. 1). (We chose to evaluate $X_{v}$ from $M_{\mathrm{B}}$ rather than $L_{115}$ because most galaxies in the sample exhibit more accurate measurements of $M_{\mathrm{B}}$.) Our sample covers a wide range of galaxy types, masses, and environments, and has $49 \%$ overlap with the subsample of the FCRAO Extragalactic CO Survey used for the CO-LF by Keres et al. (see prev. Sect.). We deliberately limited the overlap of the two samples to 50\%, in order to control sample biases.

This sample exhibits clear correlations found between $\eta_{\text {gal }}$ and $T$ and cold gas mass $\mathrm{M}_{\mathrm{g}}$, and we shall introduce three models based on these correlations. Model 1 is galaxy type dependent, as suggested by Fig. 3 (left) and earlier studies [e.g. 4, 5]. The ratio $\eta_{\text {gal }}$ increases by roughly an order of magnitude when passing from late-type spirals (Scd-Sd) to early-type spirals and lenticulars (S0-S0/a) - a trend that can be explained by the higher gas pressure in the bulge component [27]. The monotonous trend seems to break down between lenticular and elliptical galaxies, where the physical situation becomes more complex. In fact, many ellipticals comprise molecular gas in their center with no detectable HI-counterpart (e.g. Virgo cluster ellipticals), while others exhibit HI-dominated outer regions left over by mergers. To tackle the different behavior of ellipticals and spirals we chose a piecewise linear relation with different parameters for the two populations,

$$
\log \left(\eta_{\text {gal }, 1}\right)=\left\{\begin{array}{ll}
c_{0}^{e l}+c_{1}^{e l} T & \text { if } T<T_{0} \\
c_{0}^{s p}+c_{1}^{s p} T & \text { if } T \geq T_{0}
\end{array}\right\}+\sigma_{\text {phy }}
$$

where $c_{0}^{e l}, c_{1}^{e l}, c_{0}^{s p}, c_{1}^{s p}$ are free parameters to be fitted to the data, and $T_{0}$ is at the intersection of the two straight lines, i.e. $c_{0}^{e l}+c_{1}^{e l} T_{0} \equiv c_{0}^{s p}+c_{1}^{s p} T_{0} . \sigma_{\text {phy }}$ denotes an estimation of the true physical scatter.

Model 2 addresses the correlation between $\eta_{\mathrm{gal}}$ and $\mathrm{M}_{\mathrm{g}}$. Motivated by the roughly linear correlation in our sample, we chose the form

$$
\log \left(\eta_{\text {gal }, 2}\right)=k_{0}+k_{1} \log \left(\mathfrak{m}_{\mathrm{g}}\right)+\sigma_{\text {phy }}
$$

where $\mathfrak{m}_{\mathrm{g}} \equiv \mathrm{M}_{\mathrm{g}} /\left[10^{9} h^{-2} \mathrm{M}_{\odot}\right]$, and $k_{0}, k_{1}$ are free parameters.

In principle, the type-dependent model 1 and the cold gas mass-dependent model 2 could be different manifestations of the same relation, if they are related via a typedependence of the average cold gas mass. To lift a possible degeneracy, and because 

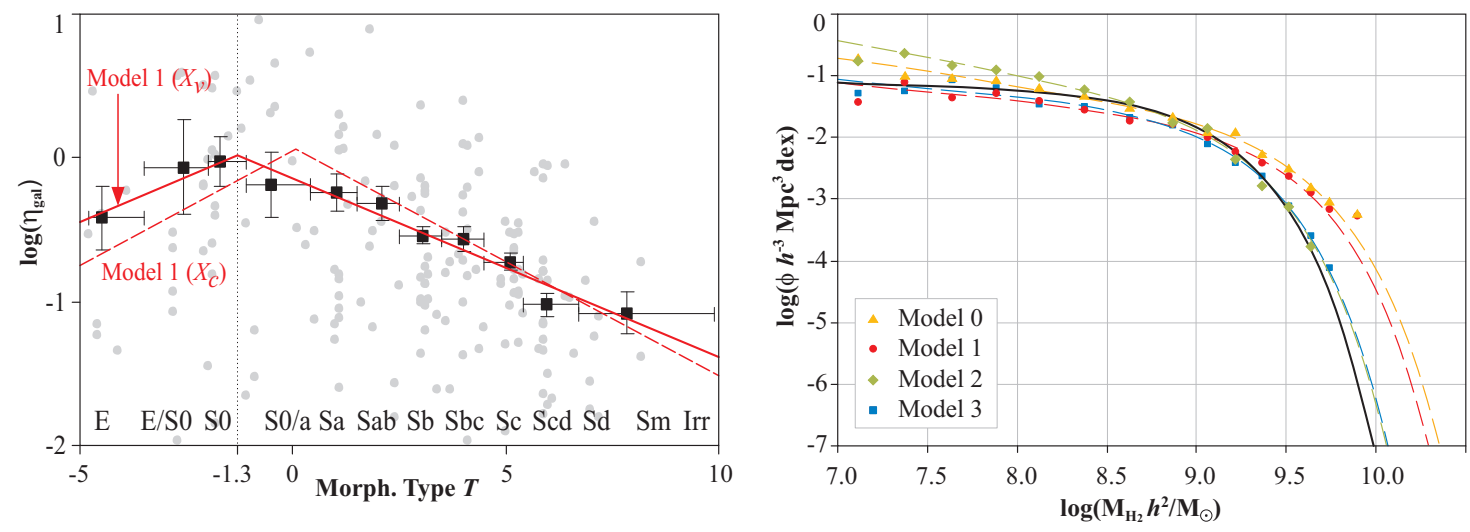

FIGURE 3. LEFT: Mass ratio versus numerical Hubble type. (dots) data points obtained via the variable factor $X_{v}$ with scatter; (black points) binned data, vertical bars are statistical uncertainties obtained via bootstrapping, horizontal bars are the bin intervals; (solid line) model 1 fitted to the data points [not to the bins]; (dashed line) model 1 fitted to the undisplayed data points for the constant factor $X_{c}$. RIGHT: (various symbols) $\mathrm{H}_{2}$-MFs constructed from the HIPASS HI-catalog using the different HI-to$\mathrm{H}_{2}$ conversion models derived in $\S 3.1$, (dashed lines) Schechter fits, (black solid line) Schechter function matching the $\mathrm{H}_{2}-\mathrm{MF}$ derived from the CO-LF, also shown in Fig. 2 (right). All functions correspond to models with variable conversion $X_{v}$.

a simultaneous dependence on two galaxy properties is suggested by the analytical derivation in [27], we shall introduce the bilinear model 3,

$$
\log \left(\eta_{\mathrm{gal}, 3}\right)=\left\{\begin{array}{c}
c_{0}^{e l}+c_{1}^{e l} T\left(<T_{0}\right) \\
c_{0}^{s p}+c_{1}^{s p} T\left(\geq T_{0}\right)
\end{array}\right\}+k_{1} \log \left(\mathrm{M}_{\mathrm{g}}\right)+\sigma_{\mathrm{phy}}
$$

where $c_{0}^{e l}, c_{1}^{e l}, c_{0}^{s p}, c_{1}^{s p}, k_{1}$ are free parameters and $c_{0}^{e l}+c_{1}^{e l} T_{0} \equiv c_{0}^{s p}+c_{1}^{s p} T_{0}$.

To compare the three models with a constant molecular fraction, such as often used in the literature, we finally introduce a constant model $0, \log \left(\eta_{\mathrm{gal}, 0}\right)=c_{0}+\sigma_{\mathrm{phy}}$.

The free parameters were determined by minimizing the rms-deviation of $\log \left(\eta_{\text {gal }}\right)$ from the model predictions. Optimization in log-space is the only reasonable choice, since $\eta_{\text {gal }}$ is subject to Gaussian scatter in log-space. Therefore, only models fitted in log-space can be ascribed simple Gaussian scatter, whereas other fits require more complex distributions. The most probable values and likelihoods of all parameters are summarized in Table 2 for both conversion factors $X_{v}$ and $X_{c}$. Our estimation of the true physical scatters $\sigma_{\text {phy }}$ is explained in [27], but we already note that the generally smaller scatter in case of a variable conversion factor $X_{v}$ supports the variable $\mathrm{H}_{2}-\mathrm{CO}$ conversion against a constant one.

\subsection{Recovering the $\mathrm{H}_{2}$-MF from HI-data}

Applying the above models of $\eta_{\text {gal }}$, we recovered the $\mathrm{H}_{2}$-MF from the HI-data in the HIPASS catalog (see Fig. 3, right). For comparison, the plot also includes the $\mathrm{H}_{2}-\mathrm{MF}$ derived from the CO-LF using the same conversion factor (black solid line). Clearly, model 3 provides the best simultaneous agreement for small and high masses. Model 1 
TABLE 2. Most likely parameters for the four models of $\eta_{\mathrm{gal}}$.

\begin{tabular}{|c|cc|cc|}
\hline & \multicolumn{2}{|c|}{ variable $\mathbf{X}_{\mathbf{v}}$} & \multicolumn{2}{c|}{ constant $\mathbf{X}_{\mathbf{c}}$} \\
\hline $\mathbf{0}$ & $c_{0}=-0.58_{-0.23}^{+0.16}$ & $\sigma_{\text {phy }}=0.39$ & $c_{0}=-0.50_{-0.23}^{+0.16}$ & $\sigma_{\text {phy }}=0.44$ \\
\hline $\mathbf{1}$ & $c_{0}^{e l}=+0.18_{-0.22}^{+0.40}$ & $c_{0}^{s p}=-0.14_{-0.07}^{+0.10}$ & $c_{0}^{e l}=+0.06_{-0.12}^{+0.28}$ & $c_{0}^{s p}=+0.07_{-0.17}^{+0.11}$ \\
& $c_{1}^{e l}=+0.12_{-0.05}^{+0.14}$ & $c_{1}^{s p}=-0.12_{-0.02}^{+0.01}$ & $c_{1}^{e l}=+0.16_{-0.04}^{+0.07}$ & $c_{1}^{s p}=-0.16_{-0.03}^{+0.03}$ \\
& $T_{0}=-1.3_{-0.5}^{+1.2}$ & $\sigma_{\text {phy }}=0.27$ & $T_{0}=0.0_{-0.4}^{+0.4}$ & $\sigma_{\text {phy }}=0.33$ \\
\hline $\mathbf{2}$ & $k_{0}=-0.51_{-0.04}^{+0.03}$ & $\sigma_{\text {phy }}=0.30$ & $k_{0}=-0.53_{-0.04}^{+0.03}$ & $\sigma_{\text {phy }}=0.42$ \\
& $k_{1}=-0.24_{-0.05}^{+0.05}$ & & $k_{1}=0.00_{-0.07}^{+0.05}$ & \\
\hline $\mathbf{3}$ & $c_{0}^{e l}=-0.01_{-0.16}^{+0.25}$ & $c_{0}^{s p}=-0.02_{-0.09}^{+0.10}$ & $c_{0}^{e l}=+0.05_{-0.21}^{+0.26}$ & $c_{0}^{s p}=+0.05_{-0.21}^{+0.10}$ \\
& $c_{1}^{s p}=-0.13_{-0.02}^{+0.02}$ & $c_{1}^{e l}=+0.13_{-0.04}^{+0.07}$ & $c_{1}^{e l}=+0.14_{-0.05}^{+0.11}$ & $c_{1}^{s p}=-0.16_{-0.02}^{+0.02}$ \\
& $k_{1}=-0.18_{-0.07}^{+0.06}$ & $T_{0}=-0.1_{-0.6}^{+1.2}$ & $k_{1}=+0.06_{-0.11}^{+0.11}$ & $T_{0}=-0.00_{-0.5}^{+1.5}$ \\
& $\sigma_{\text {phy }}=0.15^{-0.03}$ & $\sigma_{\text {phy }}=0.33$ & \\
\hline
\end{tabular}

over-estimates the density of heavy $\mathrm{H}_{2}$-masses, probably by over-estimating $\eta_{\text {gal }}$ of gasrichest early-type spiral galaxies. The latter have a low molecular fraction (see model 2), but they are a minority within otherwise gas-poor but molecule-rich early-type spirals. While model 2 overcomes this issue and produces the right density of heavy $\mathrm{H}_{2}$-masses, it fails by a factor 3-4 in the low-mass end $\left(\mathrm{M}_{\mathrm{H}_{2}} \lesssim 10^{8} \mathrm{M}_{\odot}\right)$. This is a direct manifestation of assigning high molecular fractions to all gas-poor galaxies, which neglects small young spirals with a dominant atomic phase. Finally, model 0 seems to suffer from similar limitations in both ends of the $\mathrm{H}_{2}-\mathrm{MF}$.

The increasing mutual agreement between the CO-LF-based $\mathrm{H}_{2}-\mathrm{MF}$ and HI-based $\mathrm{H}_{2}$-MFs with increasing model complexity is an indicator for the good quality of the $\mathrm{H}_{2}-$ MF derived from the CO-LF. With regard to the unknown completeness, Keres et al. [2] confirmingly argued that their CO-LF does not substantially suffer from incompleteness by analyzing the FIR-LF produced from the same sample. The surprisingly strong agreement of model 3 in particular supports this mass- and type-dependent model against models depending on one parameter only. This affirmation will be strongly supported by our analytical derivation of $\eta_{\text {gal }}$ in [27].

\section{OUTLOOK}

A detailed analysis of the ratio $\eta_{\mathrm{gal}}$ is presented in forthcoming papers. This will include

- Discussion of the scatter of our phenomenological models of $\eta_{\mathrm{gal}}$.

- Analytical derivation of the $\eta_{\text {gal }}$ based on the $\eta$-pressure relation [35].

- Local cold gas-MF $\left(\mathrm{HI}+\mathrm{H}_{2}+\mathrm{He}\right)$ derived from the HIPASS data using our best model of $\eta_{\text {gal }}$.

- $\mathrm{HI}$ and $\mathrm{H}_{2}$ in cosmological simulations: application of our best model of $\eta_{\text {gal }}$ to simulate HI- and CO-emission lines based on simulated galaxy catalogs.

- Extended discussion of the cosmological evolution of $\eta_{\mathrm{gal}}, \Omega_{\mathrm{HI}}$ and $\Omega_{\mathrm{H}_{2}}$. 


\section{REFERENCES}

1. A. Boselli, J. Lequeux, and G. Gavazzi, Astron. Astrophys. 384, 33-47 (2002), arXiv : astro-ph/ 0112275.

2. D. Keres, M. S. Yun, and J. S. Young, Astrophys J. 582, 659-667 (2003), arXiv:astro-ph/ 0209413.

3. M. Fukugita, C. J. Hogan, and P. J. E. Peebles, Astrophys J. 503, 518-+ (1998), arXiv: astro-ph/9712020.

4. J. S. Young, and P. M. Knezek, Astrophys J. Lett. 347, L55-L58 (1989).

5. S. Sauty, F. Casoli, A. Boselli, M. Gerin, J. Lequeux, J. Braine, G. Gavazzi, J. Dickey, I. Kazès, and P. Fouqué, Astron. Astrophys. 411, 381-390 (2003).

6. M. A. Zwaan, M. J. Meyer, L. Staveley-Smith, and R. L. Webster, Mon. Not. R. Astron. Soc. 359, L30-L34 (2005), arXiv:astro-ph/ 0502257.

7. R. G. Bower, A. J. Benson, R. Malbon, J. C. Helly, C. S. Frenk, C. M. Baugh, S. Cole, and C. G. Lacey, Mon. Not. R. Astron. Soc. 370, 645-655 (2006), arXiv: astro-ph / 0511338.

8. G. De Lucia, and J. Blaizot, Mon. Not. R. Astron. Soc. 375, 2-14 (2007), arXiv:astro-ph/ 0606519.

9. K. M. Ferrière, Reviews of Modern Physics 73, 1031-1066 (2001), arXiv : astro-ph/ 0106359.

10. W. F. Wall, Revista Mexicana de Astronomia y Astrofisica 42, 117-126 (2006).

11. C. D. Wilson, and N. Scoville, Astrophys J. 363, 435-450 (1990).

12. J. S. Young, and N. Z. Scoville, Annu. Rev. Astron. Astrophys. 29, 581-625 (1991).

13. W. Wild, A. I. Harris, A. Eckart, R. Genzel, U. U. Graf, J. M. Jackson, A. P. G. Russell, and J. Stutzki, Astron. Astrophys. 265, 447-464 (1992).

14. M. Guelin, R. Zylka, P. G. Mezger, C. G. T. Haslam, E. Kreysa, R. Lemke, and A. W. Sievers, Astron. Astrophys. 279, L37-L40 (1993).

15. M. Guelin, R. Zylka, P. G. Mezger, C. G. T. Haslam, and E. Kreysa, Astron. Astrophys. 298, L29+ (1995).

16. S. D. Hunter, D. L. Bertsch, J. R. Catelli, T. M. Dame, S. W. Digel, B. L. Dingus, J. A. Esposito, C. E. Fichtel, R. C. Hartman, G. Kanbach, D. A. Kniffen, Y. C. Lin, H. A. Mayer-Hasselwander, P. F. Michelson, C. von Montigny, R. Mukherjee, P. L. Nolan, E. Schneid, P. Sreekumar, P. Thaddeus, and D. J. Thompson, Astrophys J. 481, 205-+ (1997).

17. N. Arimoto, Y. Sofue, and T. Tsujimoto, Pub. Astron. Soc. Japan 48, 275-284 (1996).

18. G. Paturel, C. Petit, P. Prugniel, G. Theureau, J. Rousseau, M. Brouty, P. Dubois, and L. Cambrésy, Astron. Astrophys. 412, 45-55 (2003).

19. M. Rubio, G. Garay, J. Montani, and P. Thaddeus, Astrophys J. 368, 173-177 (1991).

20. J. S. Young, S. Xie, J. D. P. Kenney, and W. L. Rice, Astrophys J. Suppl. 70, 699-722 (1989).

21. M. H. Heyer, T. M. Dame, and P. Thaddeus, "The molecular gas component of M31 and the Milky Way," in Proceedings 232. WE-Heraeus Seminar, edited by E. M. Berkhuijsen, R. Beck, and R. A. M. Walterbos, 2000, pp. 29-36.

22. A. Leroy, A. Bolatto, F. Walter, and L. Blitz, Astrophys J. 643, 825-843 (2006), arXiv: astro-ph/0602056.

23. L. J. Sage, Astron. Astrophys. 272, 123-+ (1993).

24. M. H. Heyer, E. Corbelli, S. E. Schneider, and J. S. Young, Astrophys J. 602, 723-729 (2004), arXiv:astro-ph/0311226.

25. T. M. Dame, D. Hartmann, and P. Thaddeus, Astrophys J. 547, 792-813 (2001), arXiv: astro-ph/0009217.

26. J. S. Young, S. Xie, L. Tacconi, P. Knezek, P. Viscuso, L. Tacconi-Garman, N. Scoville, S. Schneider, F. P. Schloerb, S. Lord, A. Lesser, J. Kenney, Y.-L. Huang, N. Devereux, M. Claussen, J. Case, J. Carpenter, M. Berry, and L. Allen, Astrophys J. Suppl. 98, 219-+ (1995).

27. D. Obreschkow et al., (forthcoming) (2008).

28. G. A. Welch, and L. J. Sage, Astrophys J. 584, 260-277 (2003), arXiv: astro-ph/ 0210337.

29. L. J. Sage, and G. A. Welch, Astrophys J. 644, 850-861 (2006).

30. L. D. Matthews, Y. Gao, J. M. Uson, and F. Combes, Astron. J. 129, 1849-1862 (2005), arXiv: astro-ph/0501359.

31. A. Georgakakis, A. M. Hopkins, A. Caulton, T. Wiklind, A. I. Terlevich, and D. A. Forbes, Mon. Not. R. Astron. Soc. 326, 1431-1440 (2001), arXiv: astro-ph/ 0105435. 
32. P. Andreani, F. Casoli, and M. Gerin, Astron. Astrophys. 300, 43-+ (1995), arXiv: astro-ph/ 9501014.

33. J. F. Lees, G. R. Knapp, M. P. Rupen, and T. G. Phillips, Astrophys J. 379, 177-215 (1991).

34. H. A. Thronson, Jr., L. Tacconi, J. Kenney, M. A. Greenhouse, M. Margulis, L. Tacconi-Garman, and J. S. Young, Astrophys J. 344, 747-762 (1989).

35. L. Blitz, and E. Rosolowsky, Astrophys J. 650, 933-944 (2006), arXiv: astro-ph/ 0605035. 